\title{
Simultaneous development of adenocarcinoma and gastrointestinal stromal tumor (GIST) in the stomach: case report
}

\author{
Daigo Yamamoto ${ }^{1,2^{*}}$, Yoshinori Hamada', Yu Tsubota ${ }^{1}$, Katsuhiro Kawakami ${ }^{3}$, Chizuko Yamamoto ${ }^{4}$ and \\ Mitsuo Yamamoto ${ }^{2}$
}

\begin{abstract}
Background: Gastrointestinal stromal tumors (GISTs) and adenocarcinoma are distinct neoplasms originating from different cell layers. Approximately 20\% of patients with GIST develop other cancers.

Case presentation: We report a case of the coexistence of adenocarcinoma and gastrointestinal stromal tumor (GIST). Gastric endoscopy showed the ulcerated tumor with bleeding along the lesser curvature of the proximal stomach and a submucosal nodule that measured about $3 \mathrm{~cm}$ in diameter in the lower part of the stomach body. Their pathological examination showed gastric cancer (poorly differentiated diffuse adenocarcinoma) and GIST (low-risk category). Further, immunohistochemical staining for C-kit and CD34 was positive, while that for SMA and S-100 was negative.
\end{abstract}

Conclusion: Although it is not easy to speculate on the coexistence of adenocarcinoma and GIST, pre-and postoperative diagnoses may be essential, and such cancer development is not considered to be unusual.

Keywords: gastric cancer, GIST, stomach

\section{Background}

In recent years, the synchronous occurrence of tumors of different histotypes arising in the same organ has been reported more frequently. Gastrointestinal stromal tumors (GISTs) and adenocarcinoma are distinct neoplasms originating from different cell layers. Although adenocarcinoma constitutes the most common type of gastric tumor, the synchronous development of a GIST is relatively rare [1-6]. Here, we present and discuss a case of synchronous gastric cancer and GIST.

\section{Case presentation}

The patient was a 67-year-old man, who had been admitted to the hospital due to melena and a hematocrit level of $20.1 \%$. Nasogastric intubation revealed a fresh blood clot in the stomach. Urinalysis, chest and abdominal films, and liver function tests were within normal

\footnotetext{
* Correspondence: yamamotd@hirakata.ac.jp

'Department of Surgery, Kansai Medical University, Hirakata, Japan

Full list of author information is available at the end of the article
}

limits, and serum creatinine was $1.2 \mathrm{mg} / \mathrm{dl}$. Five units of packed blood cells were transfused promptly.

Abdominal CT scan showed a well-defined, heterogeneous mass $(3 \times 4 \mathrm{~m})$ which was located in the lower part of the stomach body (Figure 1). Gastric endoscopy showed the ulcerated tumor with bleeding along the lesser curvature of the proximal stomach (Figure 2) and a submucosal nodule that measured about $3 \mathrm{~cm}$ in diameter in the lower part of the stomach body (Figure 3). No other metastatic lesions in other organs were found on abdominal ultrasonography or the CT scan. Highgrade gastrointestinal bleeding persisted, necessitating the additional transfusion of ten units of packed red blood cells. Subsequently, the patient underwent a total gastrectomy. During the operation, there was no other evidence of metastatic disease in the intra-abdominal cavity. Macroscopic examination of the total gastrectomy specimen showed Borrman type- 2 tumor measuring $5 \times 6 \mathrm{~cm}$ and submucosal nodule measuring $3 \times 4$ $\mathrm{cm}$ in the stomach (Figure 4). On histopathological examination, the Borrman type- 2 tumor gastric tumor

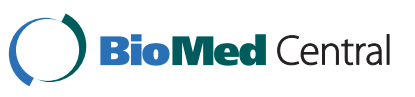




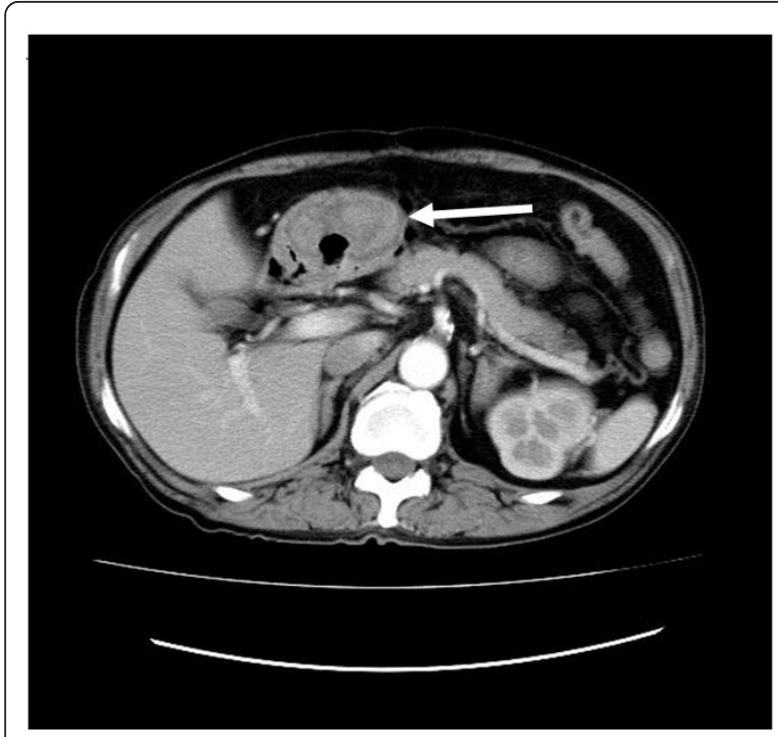

Figure 1 An abdominal computed tomography (CT) scan showing a heterogeneous mass $(3 \mathrm{~cm} \times 4 \mathrm{~cm})$ located in the lower part of the stomach body. The tumor is indicated with an arrow.

showed transmural infiltration by a poorly differentiated diffuse adenocarcinoma (Figure 5A). There was no vascular invasion and no lymph node metastasis. Further histopathological examination of the submucosal nodule revealed GIST of the low-risk category [14], which was composed of cytologically bland spindle cells and showed a low mitotic index $(<5 / 50 \mathrm{HPF})$.

The immunohistochemistry indicated strong staining for CD34 and C-kit, while expressions of SMA and S100 were negative (Figure 5B-F). These findings confirmed the simultaneous development of gastric cancer

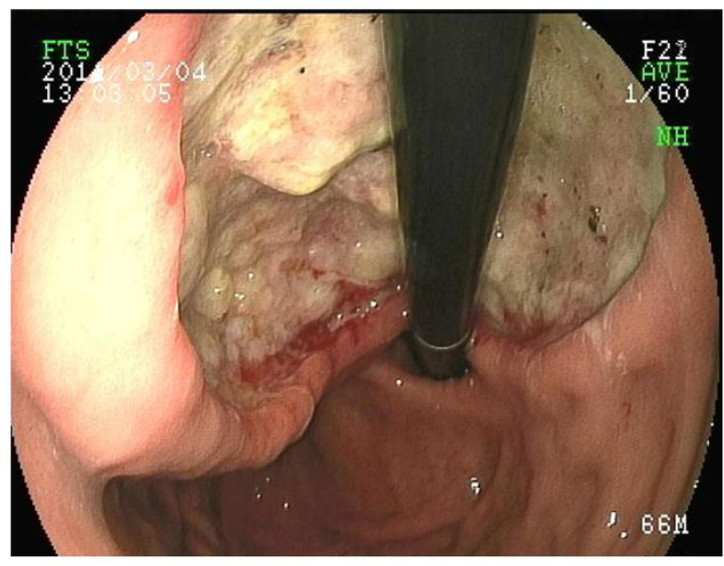

Figure 2 Gastric endoscopy confirmed an ulcerated tumor with bleeding along the lesser curvature of the proximal stomach.

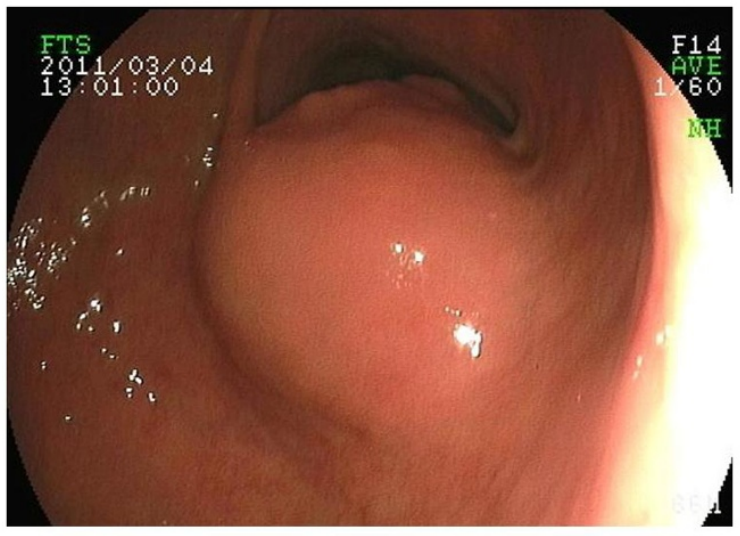

Figure 3 Gastric endoscopy showing a submucosal nodule that measured about $3 \mathrm{~cm}$ in diameter in the lower part of the stomach body.

and GIST. The patient was subsequently discharged without any complications.

\section{Discussion}

The coexistence of adenocarcinoma and GIST is uncommon, and GIST has often been detected incidentally in the gastric mucosa or serosa, or, occasionally, intramurally, at surgery [1-4]. Further, gastric tumors are derived from various other carcinomas and a precise pre- and post-operative diagnosis is important. However, when the GIST is submucosal or subserosal, the gastric mucosa may not be invaded, and endoscopic biopsies

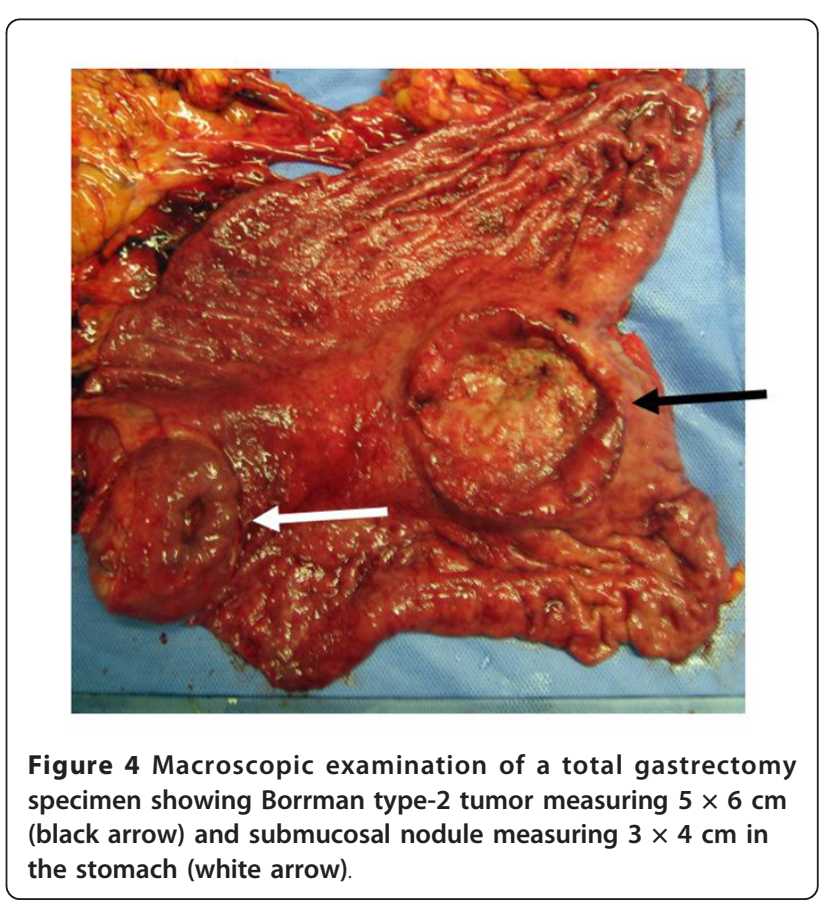



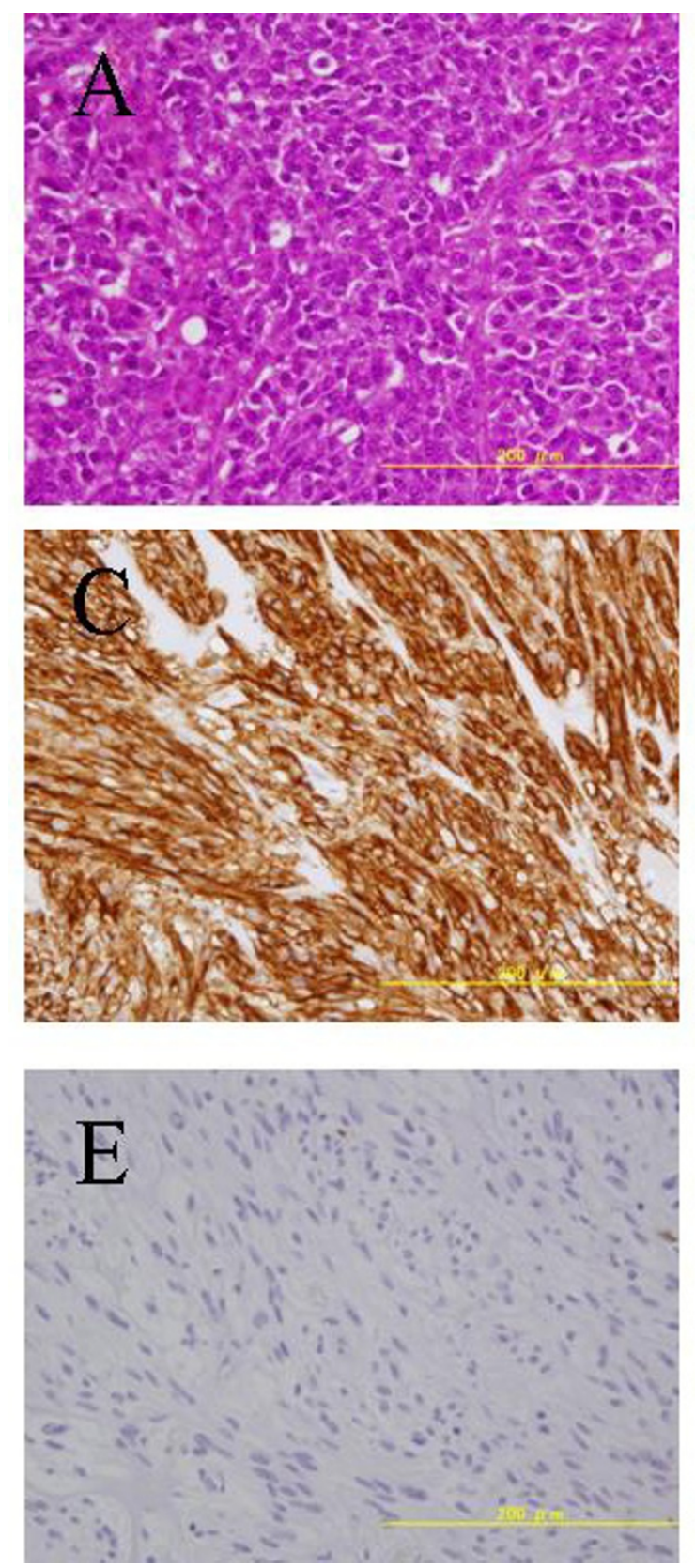
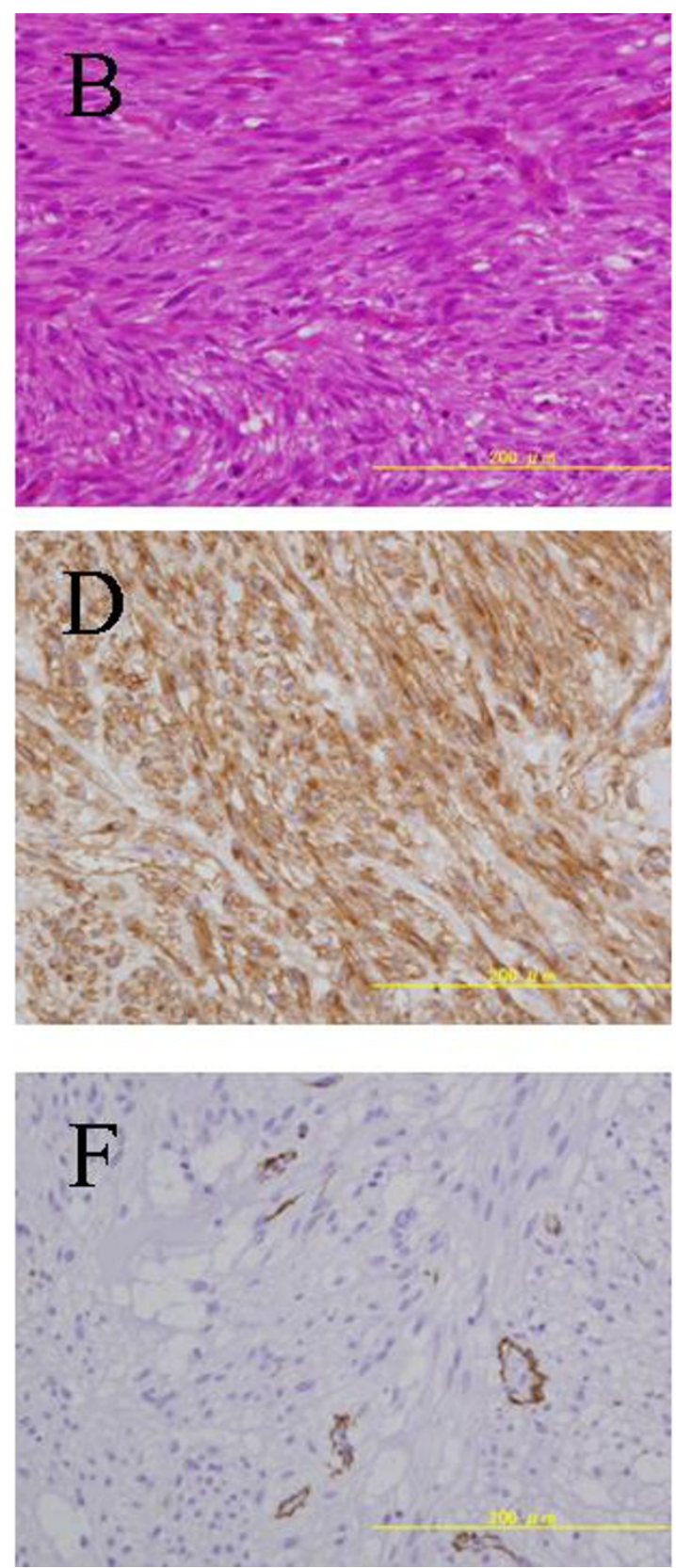

Figure 5 Microscopic examination of the proximal tumor in the stomach demonstrating typical histological findings of adenocarcinoma (A). In addition, histopathological examination of the submucosal nodule revealed GIST of the low-risk category, composed of cytologically bland spindle cells with a low mitotic index $(<5)(B)$. Immunohistochemistry indicated strong staining for CD34 (C) and C-kit (D), while negative results were observed for S-100 (E) and SMA (F).

can be normal. In fact, in our case, the pre-operative biopsy fragments showed only adenocarcinoma, and the GIST was detected only in the resected stomach. Therefore, it may be difficult to diagnose preoperatively.

Approximately $20 \%$ of patients with GIST develop other cancers and various hypotheses have been proposed regarding the simultaneous development of GIST and adenocarcinoma [7-9]. It is not clear whether this is a simple incidental coexistence or the two lesions are connected by a causal relationship. Gene mutations may underlie tumor predisposition in patients harboring a double neoplasia. However, at present, no data are available to support such a hypothesis.

GIST is the most common mesenchymal tumor [10], accounting for about 0.1 to $3 \%$ of all GI tumors [11]. IHC staining, such as is for CD34, smooth muscle actin 
(SMA), and S100, as well as c-kit (CD117), is necessary to make an accurate diagnosis of GIST [13]. It was revealed that c-kit and CD34 showed diffuse, strongly positive expressions in GIST. Rabin et al. [11] reported that 40 to $70 \%$ of GIST's were positive for CD34, 20 to $30 \%$ were positive for SMA, and 10\% were positive for S100 protein $[12,13]$. Therefore, immunostaining of CD34, c-kit, SMA, and S100 is useful, and we could confirm the histological diagnosis using these markers. Accordingly, immunohistochemical as well as clinical information may be required in order to diagnose GIST appropriately. Although it is not easy to speculate on the coexistence of adenocarcinoma and GIST, pre- and post-operative diagnoses may be essential. Further the adjuvant therapy and lymphadenectomy are important. The refinement of risk stratification systems [14] will increase the precision of these systems for predicting recurrence, which may facilitate improvements in individual disease management.

\section{Consent}

Written consent was obtained from the patient for publication of this study and the related photos.

\footnotetext{
Author details

'Department of Surgery, Kansai Medical University, Hirakata, Japan.

Department of Surgery, Seiko hospital, Neyagawa, Osaka, Japan.

${ }^{3}$ Department of Neurosurgery, Seiko hospital, Neyagawa, Osaka, Japan.

${ }^{4}$ Department of Internal Medicine, Seiko hospital, Neyagawa, Osaka, Japan.
}

\section{Authors' contributions}

The authors(s) wrote the original manuscript.

\section{Competing interests}

The authors declare that they have no competing interests.

Received: 18 August 2011 Accepted: 9 January 2012

Published: 9 January 2012

\section{References}

1. Bircan S, Candir O, Aydin S, Baspinar S, Buldul M, Kapucuoglu M, Karahan N, Ciris M: Synchronous primary adenocarcinoma and gastrointestinal stromal tumour in the stomach. a report of two cases. Turk $J$ Gastroenterol 2004, 15:187-191.

2. Rauf F, Ahmad Z, Muzzafar S, Hussaini AS: Synchronous occurrence of gastrointestinal stromal tumour and gastric adenocarcinoma. a case report. J Pak Med Assoc 2006, 56:184-186.

3. Liu SW, Chen GH, Hsieh PP: Collision tumour of the Stomach. A case report of Mixed Stromal Tumour and Adenocarcinoma. J Clin Gastroenterol 2002, 35:332-334.

4. Maiorana A, Fante R, Maria Cesinaro A, Adriana Fano R: Synchronous occurrence of epithelial and stromal tumors in the stomach. a report of 6 cases. Arch Pathol Lab Med 2000, 124:682-686.

5. Yamamoto D, Hamada Y, Okazaki S, Kawakami, Kanzaki S, Yamamoto C, Yamamoto M: Metastatic gastric tumor from renal cell carcinoma. Gastric Cancer 2009, 12:170-173.

6. Yamamoto D, Yoshida H, Sumida K, Ueyama Y, Kanematsu S, Shoji T, Sueoka N, Tanaka K, Tsubota Y, Kon M: Gastric Tumor from Metastasis of Breast Cancer. Anticancer Res 2010, 30:3705-3708.

7. Kaffes A, Hughes L, Hollinshead J, Katelaris P: Synchronous primary adenocarcinoma, mucosa-associated lymphoid tissue lymphoma and a stromal tumor in a Helicobacter pylori-infected stomach. J Gastroenterol Hepatol 2002, 17:1033-1036.

8. Fletcher CD, Berman JJ, Corless C: Diagnosis of gastrointestinal stromal tumors: a consensus approach. Hum Pathol 2002, 33:459-65.

9. Pandurengan RK, Dumont AG, Araujo DM, Ludwig JA, Ravi V, Patel S, Garber J, Benjamin RS, Strom SS, Trent JC: Survival of patients with multiple primary malignancies: a study of 783 patients with gastrointestinal stromal tumor. Ann Oncol 2010, 21:2107-11.

10. Pidhorecky I, Cheney RT, Kraybill WG, Gibbs JF: Gastrointestinal stromal tumors. current diagnosis, biologic behavior, and management. Ann Surg Oncol 2000, 7:705-12.

11. Rabin I, Chikman B, Lavy R, Sandbank J, Maklakovsky M, Gold-Deutch R: Gastrointestinal stromal tumors: a 19 year experience. Isr Med Assoc J 2009, 11:98-102

12. Liu FY, Qi JP, Xu FL, Wu AP: Clinicopathological and immunohistochemical analysis of gastrointestinal stromal tumor. World J Gastroenterol 2006, 12:4161-5.

13. Kang Na Y, Jung Ra H, Hwang I: Clinicopathological and Immunohistochemical Features of Gastointestinal Stromal Tumors. Cancer Res Treat 2010, 42:135-143.

14. Patel S: Navigating risk stratification systems for the management of patients with GIST. Ann Surg Oncol 2011, 18:1698-704.

doi:10.1186/1477-7819-10-6

Cite this article as: Yamamoto et al:: Simultaneous development of adenocarcinoma and gastrointestinal stromal tumor (GIST) in the stomach: case report. World Journal of Surgical Oncology 2012 10:6.

\section{Submit your next manuscript to BioMed Central and take full advantage of:}

- Convenient online submission

- Thorough peer review

- No space constraints or color figure charges

- Immediate publication on acceptance

- Inclusion in PubMed, CAS, Scopus and Google Scholar

- Research which is freely available for redistribution

Submit your manuscript at www biomedcentral com/submit 